\title{
The prognosis of stage IA synchronous endometrial endometrioid and ovarian carcinomas
}

\author{
Xiangbo Zhan ${ }^{1,2} \cdot$ Lei Li $^{1}$ (1) $\cdot$ Ming Wu${ }^{1} \cdot$ Jinghe Lang ${ }^{1}$
}

Received: 26 April 2019 / Accepted: 3 September 2019 / Published online: 14 September 2019

(C) The Author(s) 2019

\begin{abstract}
Introduction Little is known about the prevalence and prognosis of synchronous endometrial and ovarian carcinomas. This report explores the survival outcomes of synchronous stage IA endometrioid endometrial and stage IA ovarian carcinomas in a retrospective cohort study.

Methods All cases of pathological confirmed synchronous stage IA endometrial endometrioid and ovarian carcinomas from June 1, 2010, to June 1, 2017, in a teaching hospital were reviewed. Patients were followed up to February 1, 2019. Survival outcomes were compared between patients with and without synchronous carcinomas.

Results In total, 841 cases with confirmed FIGO stage IA endometrioid endometrial carcinomas were included in the study; 33 patients (3.9\%) had synchronous stage IA ovarian carcinomas, including $27(81.8 \%)$ and $6(18.2 \%)$ cases of endometrioid and mixed endometrioid/clear cell subtypes, respectively. After a median follow-up time of 56.8 months, 829 patients (97.9\%) had definitive survival outcomes. Synchronous ovarian carcinomas had no impact on disease-free, overall or cancer-specific overall survival in univariate and multivariate analyses.

Conclusion In these patients with stage IA endometrioid endometrial carcinoma, the genuine incidence of synchronous stage IA ovarian carcinoma was very low, and synchronous carcinoma had no significant effects on survival outcomes.
\end{abstract}

Keywords Endometrial carcinoma $\cdot$ Endometrioid subtype $\cdot$ Synchronous carcinomas

\begin{tabular}{|c|c|}
\hline \multicolumn{2}{|c|}{ Abbreviations } \\
\hline DFS & e-free \\
\hline $\mathrm{EC}$ & Endometrial cancer \\
\hline $\mathrm{OC}$ & Ovarian cancer \\
\hline
\end{tabular}

Electronic supplementary material The online version of this article (https://doi.org/10.1007/s00404-019-05288-5) contains supplementary material, which is available to authorized users.

Lei Li

lileigh@163.com

Ming Wu

wuming@pumch.cn

Jinghe Lang

langhh@ vip.163.com

1 Department of Obstetrics and Gynecology, Peking Union Medical College Hospital, Peking Union Medical College and Chinese Academy of Medical Science, Shuaifuyuan No. 1, Dongcheng District, Beijing 100730, China

2 Department of Obstetrics and Gynecology, The Sixth Affiliated Hospital of Guangzhou Medical University, Qingyuan People's Hospital, Qingyuan 511500, Guangdong, China
OS Overall survival

PALN Paraortic lymph node

PLN Pelvic lymph node

\section{Introduction}

Endometrial cancer (EC) is the fourth most common cancer and the sixth most common cause of cancer death in the United States [1] and the ninth most common cancer and tenth most common cause of cancer death in China [2]. Each year, there are an estimated 63.4 thousand new cases and 21.8 thousand deaths due to EC in China [2]. Despite a favorable prognosis, there is insufficient evidence to recommend screening for EC in women at average risk or in those who have an increased risk due to a history of unopposed estrogen therapy, tamoxifen therapy, late menopause, nulliparity, infertility or failure to ovulate, obesity, diabetes, or hypertension [3]. As the disease is frequently symptomatic at the early stage, EC is often diagnosed at stage I [4]. Moreover, patients with $\mathrm{EC}$ have a high incidence of other cancers. An estimated $3-5 \%$ of patients with EC also have ovarian 
cancer (OC) [5]; conversely, $10 \%$ of OCs are associated with ECs [6]. Synchronous carcinomas in the ovary and endometrium are the most frequent combination (50-70\%) among all synchronous female genital tract malignancies. There have been debates on the prognosis and treatment of these tumor types in terms of primary or metastatic carcinoma. In general, patients with synchronous endometrial and ovarian carcinomas have a better overall prognosis than do patients with single-organ cancer with ovarian or endometrial spread [7]. The median 5-year disease-free survival (DFS) rate is reported to be $65 \%$ for synchronous EC and OC but is less than $50 \%$ for stage IIIA EC with ovarian spread [8].

In this study, we compared survival outcomes of patients with stage I synchronous endometrioid EC and OC and patients with only stage I endometrioid EC. The effects of adjuvant therapy on survival in patients with synchronous carcinomas were also analyzed.

\section{Methods}

\section{Ethical approval and study design}

This was a retrospective cohort study implemented in a tertiary teaching hospital. The Institutional Review Board from the study center approved the study (No. ZS-1428). All patients provided written consent before treatment. The registration number is NCT03291275 (clinicaltrials.gov, registered on September 25, 2017).

All eligible patients with stage IA EC confirmed by comprehensive staging surgery were classified into two groups: Group A, with only endometrioid endometrial carcinoma; and Group B, with synchronous endometrial endometrioid and ovarian carcinomas. The primary objective was to determine the 5-year DFS and 5-year overall survival (OS) rates of the two groups. The secondary objective was to determine the impact of adjuvant treatment on the survival outcomes of these patients.

\section{Patient enrollment}

Detailed surgical and pathological data were collected by searching and reviewing electronic medical records from June 1, 2010, to June 1, 2017, at the study center. The inclusion criteria consisted of the following: primary endometrioid EC with or without synchronous OC; comprehensive staging surgery confirming stage IA synchronous endometrial and ovarian carcinoma; and detailed clinicopathological records available to retrospectively obtain information. The clinicopathological features examined that aided in the distinction between apparent independent primary tumors versus tumor metastases were according to the pathological criteria described by Scully et al. [9] Patients were excluded if they had non-endometrioid endometrial carcinomas, deep myometrial invasion, synchronous carcinomas of sites other than the ovary, any extrauterine metastasis or only simple hysterectomy surgery.

\section{Interventions and follow-up}

The patients consented to comprehensive staging procedures, which included hysterectomy, bilateral salpingoophorectomy, and retroperitoneal lymphadenectomy. All adjuvant therapies followed relevant contemporary guidelines and/or were at the physician's discretion. For grade 2-3 stage IA endometrial endometrioid carcinomas, utilization of brachytherapy was discussed, though no chemotherapy was recommended. For patients with stage IA ovarian carcinomas with grade $2-3$ endometrioid and clear cell subtypes, intravenous platinum-based therapy of 3-6 cycles was recommended.

All patients were followed up until February 1, 2019. Close follow-up according to our customized protocol was provided for all patients. Recurrence was validated by physical examination, imaging and/or biopsy. Sites of recurrence were divided into categories within the pelvic cavity and distant sites. Mortality was confirmed by reviewing medical records and interviews by telephone and/or email.

\section{Statistics}

Comparisons of continuous variables were conducted with parametric methods if assumptions of normal distribution were confirmed. Nonnormally distributed variables and categorical data were compared between the two groups using nonparametric tests. Survival curves were generated using the Kaplan-Meier method, and proportional hazards models were used to estimate hazard ratios and $95 \%$ confidence intervals for the effects of synchronous carcinomas and adjuvant therapy on DFS and OS. Multivariable analysis of DFS was performed with adjustment for important baseline risk factors. Unless otherwise stated, all analyses were performed with a two-sided significance level of 0.05 and conducted with the use of the software SPSS 22.0 (SPSS, Inc., Chicago, IL, USA).

\section{Results}

\section{Patients' characteristics}

The patient inclusion process is shown in Fig. 1. The baseline characteristics of the patients are summarized in Table 1. From June 1, 2010, to June 1, 2017, 841 cases with confirmed FIGO stage IA endometrioid endometrial carcinomas were included in the study, involving 808 cases (96.1\%) with only EC (Group A) and 33 cases (3.9\%) with 


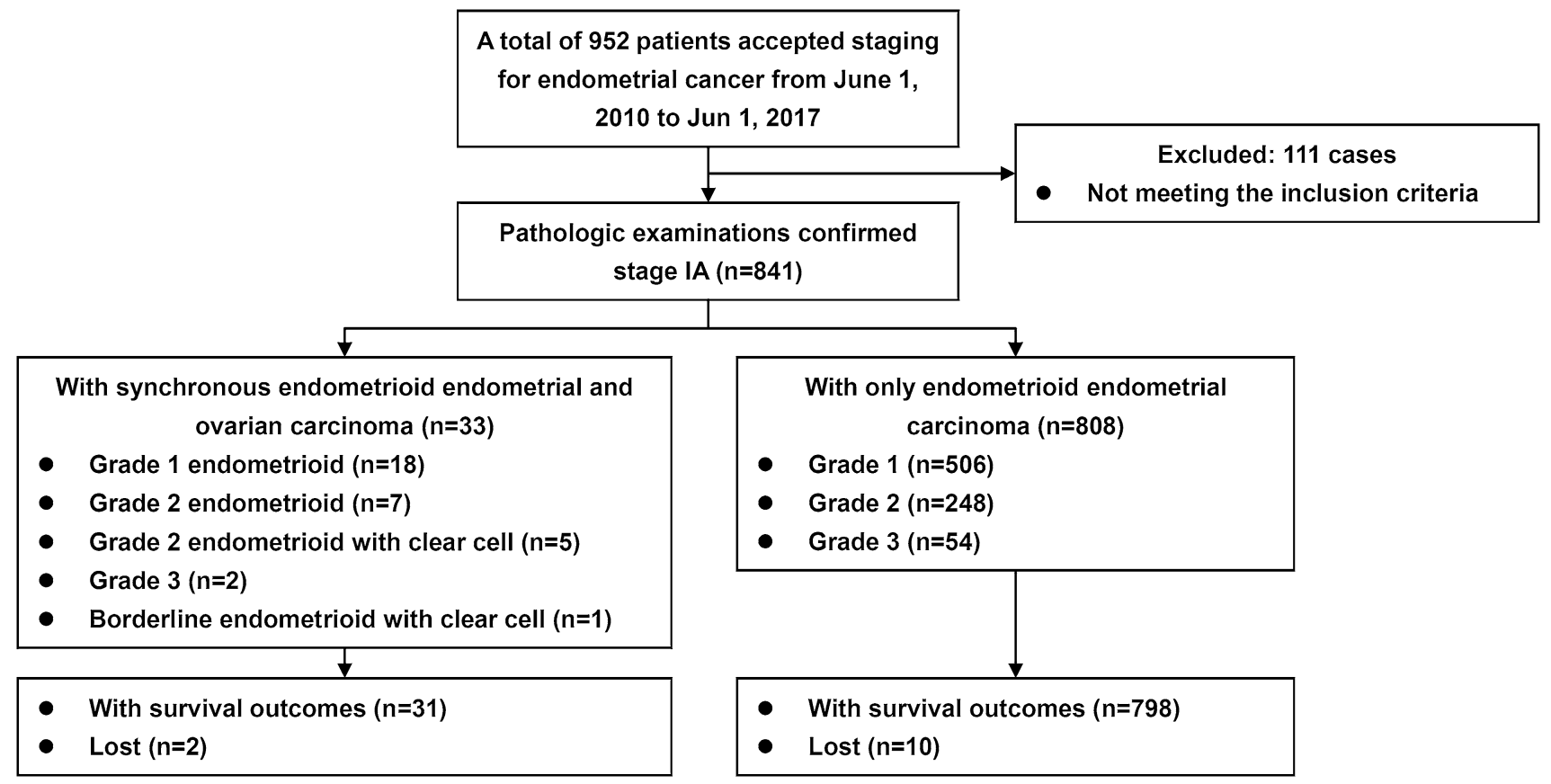

Fig. 1 Flow diagram of the study

synchronous carcinomas (Group B). Most epidemiological and clinicopathological characteristics were well balanced (Table 1). In 33 cases of mixed carcinomas, there were 29, 3 and 1 cases of concurrent endometriosis, atypical endometriosis and borderline endometrioid in the OC specimens, respectively; there were 25 and 8 cases of atypical and complex hyperplasia in the EC specimens, respectively. The subtypes of OC were as follows: 18, 7 and 3 cases of grade 1 , grade 2 and grade 3 endometrioid subtypes, respectively; 5 cases of grade 2 endometrioid with clear cell subtypes; and 1 case of borderline endometrioid with clear cell subtype.

\section{Survival outcomes of the two groups}

In total, 829 patients (97.9\%) had definitive survival outcomes with a median follow-up time of 56.5 months (range 17.8-105.4). There were 19 (2.4\%) and $2(6.5 \%)$ recurrences in the patients in Groups A and B, respectively; and there were $6(0.8 \%)$ and $1(3.2 \%)$ death, respectively. One of 7 (14.3\%) deaths was not due to EC (Supplement Table 1) and occurred in Group A.

Patients in Groups A and B had similar 5-year DFS (97\% and 94\%, $p=0.172$ ), OS (99\% and 97\%, $p=0.648$ ) and cancer-specific OS (99\% and 97\%, $p=0.085)$ rates in Kaplan-Meier analysis (Fig. 2). After adjusting for baseline factors (age, menopausal status, metabolic diseases, surgical routes, tumor limited to the endometrium, postoperative adjuvant therapy), compared with Group A, the hazard ratios for recurrence, mortality and cancer-specific mortality in Group B were 1.6 (95\% confidence interval: $0.2-10.0$, $p=0.619), 11.6(90.5-281.0, p=0.130)$ and $8.2(0.3-250.0$, $p=0.225$ ), respectively. In the Cox regression model, postoperative adjuvant therapy was the independent risk factor of DFS; no independent risk factor relevant for OS was found.

\section{Adjuvant treatment and survival outcomes of synchronous carcinomas}

For 33 patients with synchronous carcinomas, 25 underwent adjuvant therapy, including radiotherapy in 1 case and chemotherapy in 25 cases. After a median follow-up time of 56.8 months (range 21.5-83.4), adjuvant therapy had no impact on 5-year DFS, OS or cancer-specific OS rates. No significant risk factor was found to be relevant to recurrence or mortality for patients with synchronous carcinomas.

\section{Discussion}

In this report, we reveal that after a median follow-up of almost 5 years, patients with stage IA synchronous endometrial and ovarian carcinomas and patients with stage IA endometrial carcinomas had similar DFS and OS rates, even with adjustments for adjuvant therapy. These findings are consistent with the conclusions of a case-control study using the Surveillance, Epidemiology, and End Result Program 
Table 1 The epidemiological and clinicopathological characteristics of the patients

\begin{tabular}{|c|c|c|c|}
\hline & $\begin{array}{l}\text { Group A patients with endome- } \\
\text { trioid endometrial carcinoma } \\
(n=808)\end{array}$ & $\begin{array}{l}\text { Group B patients with synchronous endome- } \\
\text { trioid endometrial and ovarian carcinomas } \\
(n=33)\end{array}$ & $p$ \\
\hline Age (year), mean $\pm \mathrm{SD}$ & $53.0 \pm 9.1$ & $49.5 \pm 6.7$ & 0.028 \\
\hline Menopause, $n(\%)$ & $513(63.5 \%)$ & $12(36.4 \%)$ & 0.002 \\
\hline Metabolic disease, $n(\%)$ & $302(37.4 \%)$ & $7(21.2 \%)$ & 0.059 \\
\hline Diabetes & $137(17.0 \%)$ & $4(12.1 \%)$ & 0.466 \\
\hline Hypertension & $240(29.7 \%)$ & $5(15.2 \%)$ & 0.071 \\
\hline Hyperlipemia & $31(3.8 \%)$ & $1(3.0 \%)$ & 0.639 \\
\hline Obesity & $30(3.7 \%)$ & $1(3.0 \%)$ & 0.654 \\
\hline History of infertility, $n(\%)$ & $13(1.6 \%)$ & $2(6.1 \%)$ & 0.114 \\
\hline Smoking, $n(\%)$ & $23(2.8 \%)$ & $0(0.0 \%)$ & 0.393 \\
\hline Diagnostic methods, $n(\%)$ & & & 0.175 \\
\hline Dilation and curettage & $417(51.6 \%)$ & $21(63.6 \%)$ & \\
\hline Hysteroscopy & $391(48.4 \%)$ & $12(36.4 \%)$ & \\
\hline Surgical routs, $n(\%)$ & & & 0.075 \\
\hline Laparoscopy & $602(74.5 \%)$ & $20(60.6 \%)$ & \\
\hline Abdominal surgeries & $206(25.5 \%)$ & $13(39.4 \%)$ & \\
\hline PALN resection, $n(\%)$ & & & 0.151 \\
\hline Not done & $355(43.9 \%)$ & $9(27.3 \%)$ & \\
\hline At the level of common iliac vessel & $169(20.9 \%)$ & $8(24.2 \%)$ & \\
\hline Above the level of common iliac vessel & $284(35.1 \%)$ & $16(48.5 \%)$ & \\
\hline Ovarian preservation, $n(\%)$ & $14(1.7 \%)$ & $0(0.0 \%)$ & 0.568 \\
\hline Peritoneal cytology, $n(\%)$ & & & 0.297 \\
\hline Not done & $249(30.8 \%)$ & $13(39.4 \%)$ & \\
\hline Done & $559(69.2 \%)$ & $20(60.6 \%)$ & \\
\hline Differential of endometrioid EC, $n(\%)$ & & & 0.307 \\
\hline Grade 1 & $506(62.6 \%)$ & $22(66.7 \%)$ & \\
\hline Grade 2 & $248(30.7 \%)$ & $11(33.3 \%)$ & \\
\hline Grade 3 & $54(6.7 \%)$ & $0(0.0 \%)$ & \\
\hline $\begin{array}{l}\text { Maximun diameter of the tumor }(\mathrm{mm}) \\
\text { mean } \pm \text { SD }\end{array}$ & $24.5 \pm 19.3$ & $26.8 \pm 24.1$ & 0.491 \\
\hline Tumor limited to the endometrium, $n(\%)$ & $145(17.9 \%)$ & $10(30.3 \%)$ & 0.073 \\
\hline Positive LVSI, $n(\%)$ & $54(6.7 \%)$ & $1(3.0 \%)$ & 0.350 \\
\hline Lower uterine involvement, $n(\%)$ & $166(20.5 \%)$ & $8(24.2 \%)$ & 0.607 \\
\hline Positive peritoneal cytology, $n(\%)$ & $18 / 559(3.2 \%)$ & $2 / 20(10.0 \%)$ & 0.149 \\
\hline Harvested number of PLN, mean \pm SD & $24.0 \pm 9.7$ & $24.6 \pm 8.7$ & 0.733 \\
\hline Harvested number of PALN, mean \pm SD & $7.7 \pm 5.8$ & $8.4 \pm 4.9$ & 0.528 \\
\hline Post-operative adjuvant therapy, $n(\%)$ & $82(10.1 \%)$ & $25(75.8 \%)$ & $<0.001$ \\
\hline Post-operative radiotherapy, $n(\%)$ & $62(7.7 \%)$ & $1(3.0 \%)$ & 0.275 \\
\hline Post-operative chemotherapy, $n(\%)$ & $35(4.3 \%)$ & $25(75.8 \%)$ & $<0.001$ \\
\hline Post-operative chemotherapy protocols, $n(\%)$ & $n=35$ & $n=25$ & 0.434 \\
\hline Carboplatin + paclitaxel & $29(82.9 \%)$ & $22(88.0 \%)$ & \\
\hline Others & $6(17.1 \%)$ & $3(12.0 \%)$ & \\
\hline Post-operative chemotherapy cycles, mean \pm SD & $3.6 \pm 1.7$ & $4.2 \pm 1.6$ & 0.226 \\
\hline Recurrent sites, $n(\%)$ & $n=19$ & $n=2$ & 0.433 \\
\hline Within the pelvic cavity & $7(36.8 \%)$ & $0(0.0 \%)$ & \\
\hline Distant sites & $12(63.2 \%)$ & $2(\%)$ & \\
\hline
\end{tabular}

EC endometrial carcinoma, LVSI lymph-vascular space invasion, $P A L N$ para-aortic lymph nodes, $S D$ standard deviation 

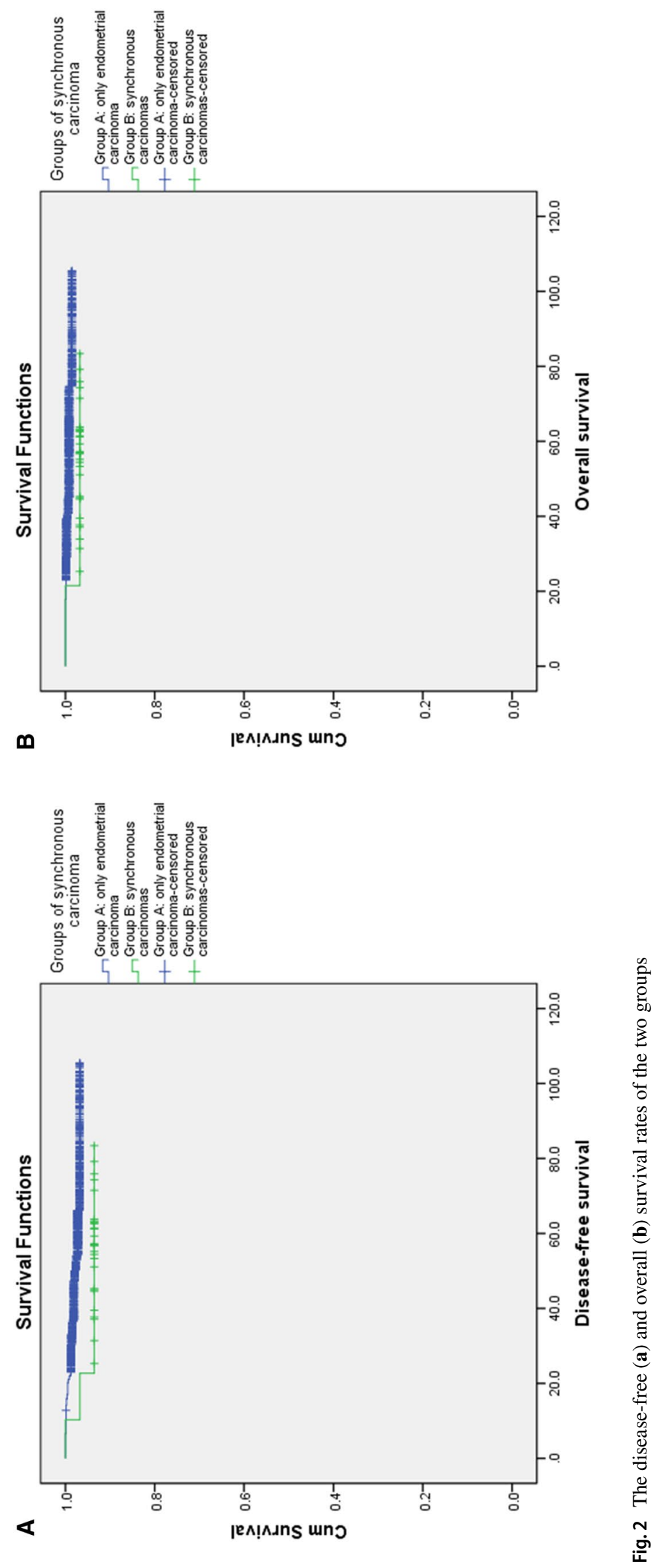
between 1973 and 2013, which suggested that the presence of synchronous OC is not associated with EC-specific survival (10-year rates $96.0 \%$ versus $95.3 \%, p=0.97)$ or $\mathrm{OS}$ (85.6\% versus $87.2 \%, p=0.10)$ [10]. Moreover, the presence of synchronous endometrioid EC does not influence the prognosis of patients with stage I OC, even in the presence of deep myometrial invasion [11]. Other authors have found that the OS rate of EC patients who had other cancers was worse than that of patients without other cancers but that disease-specific survival was not significantly different [12]. Based on these data, an appropriate prognosis could be assured within this clinical context.

In our report, all specimens of synchronous carcinomas were reviewed again to guarantee a definitive, strict diagnosis. The distinction between synchronous primary endometrial and ovarian carcinomas and metastatic malignancy is based first on histopathological criteria [9], which poses significant problems both because these criteria constitute an empiric tool based on the personal experience of the pathologist in charge and because they must account for heterogeneity among the samples analyzed [13]. A precise diagnosis is mandatory because the diagnosis is associated with a specific prognosis and needs a specific surgical and postoperative management approach, requiring extreme caution [13]. Regardless, no single criterion is perfect, and it is important to integrate all available clinicopathological, immunohistochemical and molecular data in the assessment of problematic diagnostic cases of synchronous carcinomas [14].

Whether molecular analysis can differentiate primary and metastatic carcinomas remains controversial despite several reports on synchronous carcinomas [14]. The most important limitations of these studies are the heterogeneity of tumor samples and the interpretations of findings. Synchronous gynecological carcinomas in Lynch syndrome are molecularly concordant, suggesting shared origins $[15,16]$. Based on both targeted and exome sequencing, synchronous carcinomas show evidence of a clonal relationship, suggesting that among synchronous carcinomas, disseminating cells are restricted to physically accessible and microenvironmentcompatible sites yet remain indolent, without the capacity for further dissemination [17]. These findings all suggest that synchronous carcinomas are not independent but rather represent an isolated metastatic phenomenon. However, this truly is a unique phenomenon, that is, patients with synchronous disease have a good prognosis following surgery, and systemic treatment need not be administered [18]. From a practical point of view, only conventional morphological criteria should be used for the classification (staging) of these tumors. Molecular profiling of these tumors may have prognostic and predictive meaning [19, 20].

In this study, adjuvant therapy had no significant positive impact on the survival outcomes of patients with synchronous carcinomas. According to current guidelines, observation can be provided for patients with grade 1 and grade 2 ovarian endometrioid carcinomas; intravenous platinum-based therapy can be provided for high-grade ovarian carcinoma or the clear cell subtype [21]. For stage IA endometrial endometrioid carcinomas, observation can be considered for patients with all grades [22]. To date, no consensus or guidelines have been achieved for the treatment of stage IA synchronous carcinomas. In a retrospective study in Japan, 2 of 23 patients with synchronous EC and OC experienced recurrence after adjuvant therapy, though none of 10 patients who did not receive adjuvant therapy had recurrence [23]. Studies on high-risk factors of recurrence may provide an index or indications for adjuvant therapy [24, 25]. Interestingly, concordant tumor grade was not associated with survival for stage I synchronous carcinomas in a large case-control study [10]. Due to the scarcity of synchronous carcinomas, a pooled analysis or meta-analysis likely provides a more substantial description of the role of adjuvant therapy in synchronous carcinomas.

In our study, most synchronous carcinoma patients $(60.6 \%)$ accepted open surgeries. However, the surgical approach (open versus minimally invasive surgery) for ovarian cancer has been a topic of controversy. As reported in our study, laparoscopic staging for apparent stage I epithelial ovarian cancer appeared to be as safe as laparotomy, both in the report from National Cancer Data Base [26] and in expert opinions of the German Society for Gynecologic Endoscopy [27].

The strengths of our study are the large cohort and rigorous long-term follow-up. The limitations of our study mainly involve its retrospective design, which may introduce significant observation and selection biases. The relatively small population of synchronous carcinomas limited the analysis of adjuvant treatment in terms of survival outcomes. In our retrospective cohort, $4.3 \%$ of stage IA endometrial cancer patients and $75.8 \%$ of synchronous cancer patients received adjuvant chemotherapy. As most patients had grade 1 subtypes, the high rate of chemotherapy utilization reflects an attitude of defensive medicine and the practice of overtreatment, especially for grade 1 cervical cancer patients. A well-designed prospective study should standardize the uniform treatment protocol to overcome the abovementioned bias. A lack of molecular analysis for the various components of synchronous versus metastatic carcinomas is another shortcoming. Because synchronous endometrial and ovarian neoplasia appears to be as common in younger women without obvious EC risk as in patients with possible Lynch syndrome [28], an exploration of tools for genetic analysis is also essential for the study of synchronous carcinomas. Lastly, a longer 
follow-up is essential for survival analysis of patients with mixed carcinomas.

\section{Conclusions}

In patients with stage IA endometrioid endometrial carcinoma, the appearance of synchronous stage IA ovarian carcinoma is very low and has no significant effects on survival outcomes. Adjuvant therapy does not influence the survival outcomes of this population.

\section{Compliance with ethical standards}

Conflicts of interest All authors declare that they have no conflicts of interest to disclose.

Open Access This article is distributed under the terms of the Creative Commons Attribution 4.0 International License (http://creativeco mmons.org/licenses/by/4.0/), which permits unrestricted use, distribution, and reproduction in any medium, provided you give appropriate credit to the original author(s) and the source, provide a link to the Creative Commons license, and indicate if changes were made.

\section{References}

1. Siegel RL, Miller KD, Jemal A (2019) Cancer statistics. CA Cancer J Clin 69(1):7-34

2. Chen W, Zheng R, Baade PD, Zhang S, Zeng H, Bray F et al (2016) Cancer statistics in China, 2015. CA Cancer J Clin 66(2):115-132

3. Smith RA, Andrews KS, Brooks D, Fedewa SA, ManassaramBaptiste D, Saslow D et al (2018) Cancer screening in the United States, 2018: a review of current American Cancer Society guidelines and current issues in cancer screening. CA Cancer J Clin 68(4):297-316

4. Morice P, Leary A, Creutzberg C, Abu-Rustum N, Darai E (2016) Endometrial cancer. Lancet 387(10023):1094-1108

5. AlHilli MM, Dowdy SC, Weaver AL, St Sauver JL, Keeney GL, Mariani A et al (2012) Incidence and factors associated with synchronous ovarian and endometrial cancer: a population-based case-control study. Gynecol Oncol 125(1):109-113

6. Lin KY, Miller DS, Bailey AA, Andrews SJ, Kehoe SM, Richardson DL et al (2015) Ovarian involvement in endometrioid adenocarcinoma of uterus. Gynecol Oncol 138(3):532-535

7. Chen L, Zhao Q, Lv X (2011) Characteristics and prognosis of coexisting adnexa malignancy with endometrial cancer: a single institution review of 51 cases. Arch Gynecol Obstet 283(5):1133-1137

8. Song T, Seong SJ, Bae DS, Kim JH, Suh DH, Lee KH et al (2014) Prognostic factors in women with synchronous endometrial and ovarian cancers. Int J Gynecol Cancer 24(3):520-527

9. Scully RE, Young RH, Clement PB (1998) Tumors of the ovary, maldeveloped gonads, fallopian tube, and broad ligament; Atlas of Tumor Pathology; Armed Forces Institute of Pathology: Washington, DC, USA, 1998; ISBN 978-1-881041-43-6

10. Matsuo K, Machida H, Frimer M, Marcus JZ, Pejovic T, Roman LD et al (2017) Prognosis of women with stage I endometrioid endometrial cancer and synchronous stage I endometrioid ovarian cancer. Gynecol Oncol 147(3):558-564

11. Narin MA, Karalok A, Basaran D, Ureyen I, Turkmen O, Turan T et al (2016) Does synchronous endometrioid endometrial cancer have any prognostic effect on stage I endometrioid ovarian cancer? Eur J Obstet Gynecol Reprod Biol 200:113-116

12. Tangjitgamol S, Khunnarong J, Srijaipracharoen S (2015) Synchronous and metachronous malignancy in endometrial cancer patients treated in a tertiary care center of Thailand. J Gynecol Oncol 26(4):293-302

13. Perrone AM, Girolimetti G, Procaccini M, Marchio L, Livi A, Borghese $G$ et al (2018) Potential for mitochondrial DNA sequencing in the differential diagnosis of gynaecological malignancies. Int J Mol Sci 19(7):E2048. https://doi.org/10.3390/ijms1 9072048

14. Stewart CJR, Crum CP, McCluggage WG, Park KJ, Rutgers JK, Oliva E et al (2019) Guidelines to aid in the distinction of endometrial and endocervical carcinomas, and the distinction of independent primary carcinomas of the endometrium and adnexa from metastatic spread between these and other sites. Int J Gynecol Pathol 38(Suppl 1):S75-S92

15. Niskakoski A, Pasanen A, Porkka N, Eldfors S, Lassus H, Renkonen-Sinisalo L et al (2018) Converging endometrial and ovarian tumorigenesis in Lynch syndrome: shared origin of synchronous carcinomas. Gynecol Oncol 150(1):92-98

16. Schultheis AM, Ng CK, De Filippo MR, Piscuoglio S, Macedo GS, Gatius $S$ et al (2016) Massively parallel sequencing-based clonality analysis of synchronous endometrioid endometrial and ovarian carcinomas. J Natl Cancer Inst 108(6):427

17. Anglesio MS, Wang YK, Maassen M, Horlings HM, Bashashati A, Senz J et al (2016) Synchronous endometrial and ovarian carcinomas: evidence of clonality. J Natl Cancer Inst 108(6):428

18. Dizon DS, Birrer MJ (2016) Making a difference: distinguishing two primaries from metastasis in synchronous tumors of the ovary and uterus. J Natl Cancer Inst 108(6):442

19. Hajkova N, Ticha I, Hojny J, Nemejcova K, Bartu M, Michalkova $R$ et al (2019) Synchronous endometrioid endometrial and ovarian carcinomas are biologically related: a clinico-pathological and molecular (next generation sequencing) study of 22 cases. Oncol Lett 17(2):2207-2214

20. Chao A, Wu RC, Jung SM, Lee YS, Chen SJ, Lu YL et al (2016) Implication of genomic characterization in synchronous endometrial and ovarian cancers of endometrioid histology. Gynecol Oncol 143(1):60-67

21. NCCN Clinical Practice Guidelines in Oncology (NCCN Guidelines ${ }^{\circledR}$ ). Ovarian Cancer. Including fallopian tube cancer and primary peritoneal cancer. Version 2.2018 - March 9, 2018. https ://www.nccn.org/professionals/physician_gls/pdf/ovarian.pdf. Accessed 1 Jan 2019

22. NCCN Clinical Practice Guidelines in Oncology (NCCN Guidelines ${ }^{\circledR}$ ). Uterine Neoplasms. Version 1.2019 - October 17, 2018. https://www.nccn.org/professionals/physician_gls/pdf/uteri ne.pdf. Accessed 1 Jan 2019

23. Yoneoka Y, Yoshida H, Ishikawa M, Shimizu H, Uehara T, Murakami T et al (2019) Prognostic factors of synchronous endometrial and ovarian endometrioid carcinoma. J Gynecol Oncol 30(1):e7

24. Jain V, Sekhon R, Pasricha S, Giri S, Modi KB, Shrestha E et al (2017) Clinicopathological characteristics and prognostic factors of synchronous endometrial and ovarian cancers-a single-institute review of 43 cases. Int J Gynecol Cancer 27(5):938-946

25. Solmaz U, Karatasli V, Mat E, Dereli L, Hasdemir PS, Ekin A et al (2016) Synchronous primary endometrial and ovarian cancers: a multicenter review of 63 cases. Tumori 102(5):508-513 
26. Melamed A, Keating NL, Clemmer JT, Bregar AJ, Wright JD, Boruta DM et al (2017) Laparoscopic staging for apparent stage I epithelial ovarian cancer. Am J Obstet Gynecol 216(1):50

27. Radosa JC, Radosa MP, Schweitzer PA, Juhasz-Boess I, Rimbach S, Solomayer EF (2018) Report of the survey on current opinions and practice of German Society for Gynecologic Endoscopy (AGE) members regarding the laparoscopic treatment of ovarian malignancies. Arch Gynecol Obstet 297(5):1255-1264

28. Burleigh A, Talhouk A, Gilks CB, McAlpine JN (2015) Clinical and pathological characterization of endometrial cancer in young women: identification of a cohort without classical risk factors. Gynecol Oncol 138(1):141-146

Publisher's Note Springer Nature remains neutral with regard to jurisdictional claims in published maps and institutional affiliations. 